\title{
Development of Acute Implant Peri-Implantitis After Immediate Implant Loading: A Case Report
}

\author{
Özkan $\mathrm{Y}^{\star}$ and Orbak R
}

Department of Periodontology, Ataturk University, Faculty of Dentistry, Erzurum, Republic of Turkey

*Corresponding author: Özkan Y, DDS, Research Assistant, Department of Periodontology, Ataturk University, Faculty of Dentistry, 25240, Erzurum, Republic of Turkey, E-mail: yerdaozkan@hotmail.com

Citation: Özkan Y, Orbak R (2017) Development of Acute Implant Peri-Implantitis After Immediate Implant Loading: A Case Report. J Dent Oral Care Med 3(3): 304

Received Date: June 09, 2017 Accepted Date: December 27, 2017 Published Date: December 29, 2017

\begin{abstract}
The acute apical peri-implantitis (AAPI) is defined as a clinically symptomatic periapical lesion that develops shortly after implant insertion while the coronal portion of the implant substains a normal bone-to-implant interface. The etiology of AAPI is unclear. Regardless of etiology, the management of AAPI has been attempted by either respective or regenerative techniques.

A 49-year-old patient was examined and evaluated for the right posterior immediate implant which was placed 3 weeks previously. Probing depths around the implant was normal. There wasn't bleeding on probing/mobility. The apex of the implant had radiolucent area. There was pain, swelling in right cheek. Removal of the implant was decided. BioOSS was placed in the residual bony defects and covered with a collagen barrier (Biotech membrane). After a month, in the direction of the patient's wish, a new implant was placed in mesial side of same area. Four months later, it was restored with porcelain crown.

Aim of this case report emphasizes is that different areas of the extraction region may react differently to the treatment of implants.
\end{abstract}

Keywords: Bone Regeneration; Dental Implants; Infection; Peri-implantitis

\section{Introduction}

Implant periapical lesion was first described by McAllister in 1992 [1]. This pathologies are the infectious-inflammatory process of the tissues surrounding in the apical region of the implant [2]. According to Quirynen et al. [3], peri-implantitis lesions are as a clinically symptomatic periapical lesion that develops shortly after implant insertion, whereas the bone of the coronal portion of the implant has not infection.

In generally, Implant peri-apical lesions have two basic types: acute apical peri-implantitis (non-suppurated and suppurated) and subacute (or suppurated-fistulized) apical periimplantitis.

In etiology of peri-implantitis lesions, there are different factors such as contamination of the implant and socket surface, overheating of bone during drilling, overloading of the implant, preparation of a longer implant bed than the implant itself, preexisting bone disease, implant placement in poor quality bone, presence of residual root fragments or foreign bodies, placed implant after extract a teeth with history of infection or socket decontamination might be poorly, and implant placement in proximity to an infected maxillary sinus [4-8]. Regardless of etiology, the management of AAPI has been attempted by either respective or regenerative techniques $[9,10]$.

Actually, In regions of bone have endodontic or periodontal infection, immediate implants applications have high survival rates. Villa and Rangert [11] found 97.4\% survival rates for implants which is placed immediately regions of tooth have endodontic or periodontal lesions or root fracture. In another research, Crespi et al. [12] researched that relation between asymptomatic apical periodontitis and immediately implant. They reported a survival rate of $100 \%$ for implant placed to asymptomatic apical periodontitis of area.

The incidence of this pathology is low. Reiser and Nevins [13] found 10 cases in 3800 implants placed (0.26\%); Quirynen et al. [3], in a retrospective study involving 539 implants, obtained a incidence of $1.6 \%$ in the maxilla and $2.7 \%$ in the mandible, diagnosing all cases before the second stage surgery.

The increasing popularity of implants has led to a considerable increase in the incidence of implant periapical lesions [14]. 
Diagnosis of implant periapical lesions is clinic and radiographic. But, implant periapical lesion may often be the cause of early failures, as this pathology is difficult to diagnose and it has been rarely studied in the literature.

Aim of this case report emphasizes is that different areas of the extraction region may react differently to the treatment of implants.

\section{Case report}

49-year-old patient with no systemic disease was admitted to our clinic with perio-endo lesion in right first molar tooth in mandible (\#46, FDI). In intraoral evaluation, there is a full crown on the tooth. This tooth had pain in percussion.

In radiological examination, there was radiotransparency in roots and furcation (Figure 1). Options and risks were explained to patient. The patient opted for extraction and immediate implant treatment.

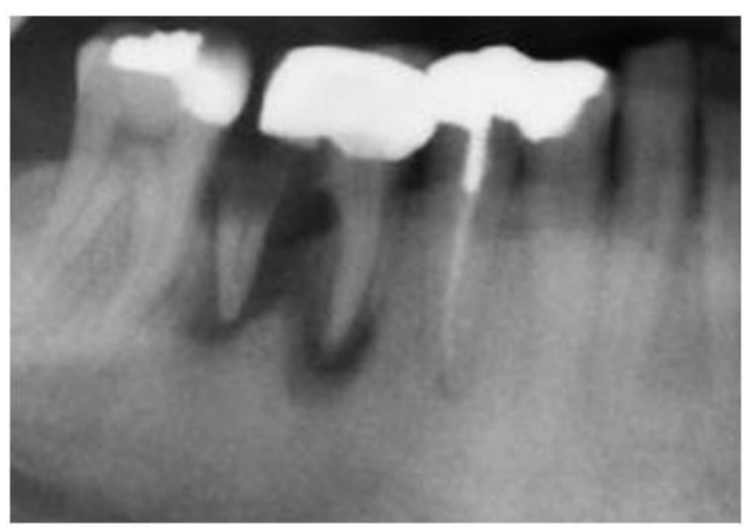

Figure 1: Failed endodontic treatment of tooth 46

Local anesthetic (20 ml Prilokain \% 2) was infiltrated and the tooth was atraumatically extracted and the socket was curetted. In distal root region of tooth, the bone volume was good for implant treatment. A full-thickness flap was raised and an osteotomy prepared by first marking the site with a \#6 round burr, then drilling the site in sequence with 2-mm, 2.8- $\mathrm{mm}$, and 3.5-mm drills. All these were internally irrigated with normal saline. All drills were used at $800 \mathrm{rpm}$. Misch bone type D-2 was encountered [15]. A 3.75-mm diameter and 11.5-mm length (Tidal Spiral Implant, Implant Innovations Inc, The Picasso Building, Caldervale Road, Wakefield, West Yorkshire) was placed into the full length of the osteotomy (Figure 2). The insertion torque was more than $35 \mathrm{Ncm}$. The site was primarily closed with 4-0 Vicryl (Ethicon). Postoperative was given antibiotics (Amoxicillin/Clavulanate potassium $1000 \mathrm{mg}$ tablets BID) and NSAI drug (Naproxen sodium $550 \mathrm{mg}$ tablets) every 12 hours for 5 days and a chlorhexidine oral rinse (Klorhex). The sutures were removed 1 week later, and the site had healed well. The patient was reappointed in 6 months for evaluation of the site.

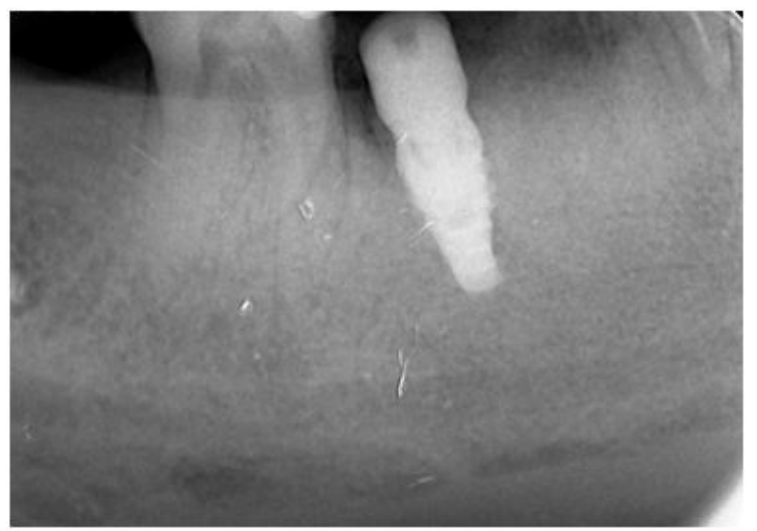

Figure 2: Implant in distal root region of tooth 46 site

Three weeks postoperatively, the patient was seen on an emergency basis for pain at the implant site and there was slightly swelling in right cheek (Figure 3). In periapical radiography, there was periapical radiolucency on bottom of the implant (Figure 4). Same antibiotics and NSAI prescribed for 7 days.

Five days later, the patient was seen for definitive treatment. A draining sinus tract was noted at the apical area of the implant. The implant was immobile, and there was no pocketing. But extraction of implant was decided. The area was locally anesthetized (20 ml Prilokain \% 2), and a full-thickness creastal flap was raised. There was a connection between infection and soft tissue by a bone-window. The apical lesion region was debrided and curetted. Removal of the implant was decided, because there was a serious bone loss due to the infection. BioOSS (Geistlich Pharma, Wolhusen, Switzerland) was placed in the residual bone defect, to protect the bone width in the area of infection, and covered with a collagen barrier (Biotech membran, Datum Biotech Ltd, Israel). Primary closure was obtained with 4-0 Vicryl. 


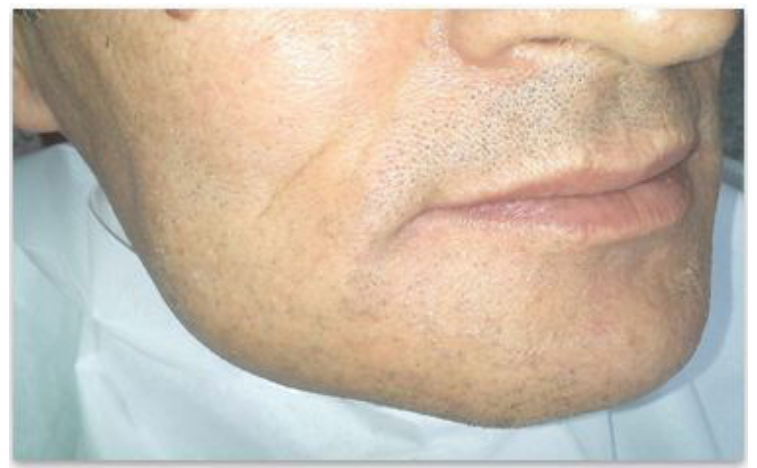

Figure 3: Slightly swelling in right cheek of patient

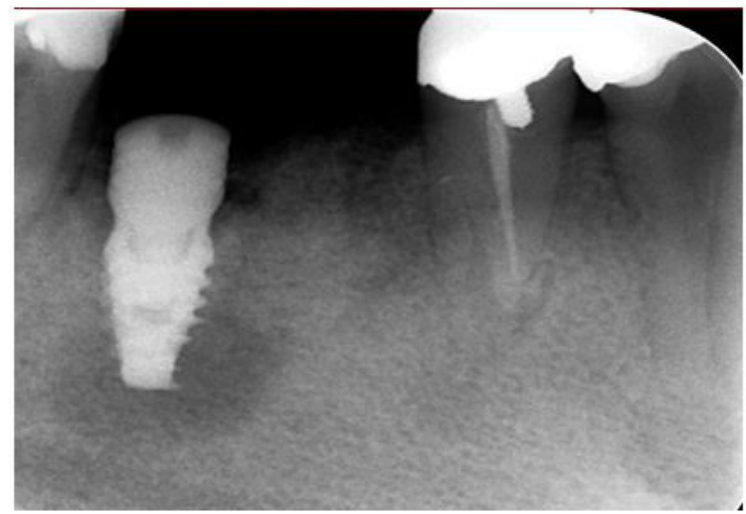

Figure 4: Apical peri-implantitis lesion

After a month, a new implant was placed in mesial location, our prosthetic plan included single-tooth implant, so we didn't need to change prosthetic plan. At 1 week, the sutures were removed and the patient stated that he had no pain or discomfort. No membrane and graft was used, because region of mesial didn't have bone loss. Primary closure was obtained with 4-0 Vicryl. At 1 week, the sutures were removed and the patient stated that he had no pain or discomfort.

4 months post implant placement, he was seen and reported no symptoms and, in fact, "felt great." At that time, a straight abutment was placed, the retaining screw tightened to $35 \mathrm{Ncm}$ and slightly prepared and impressed for a full crown cemented restoration. A provisional acrylic crown was placed. A porcelain-fused to noble alloy crown was cemented 6 weeks later and made to avoid any centric or eccentric contacts, as was the position of the natural tooth. The crown was cemented with zinc oxide and eugenol paste (Opotow) and then, 4 months later, with zinc oxide and eugenol cement. No signs or symptoms of infection were noted. A panoramic radiograph showed radiographic resolution of the apical lesion (Figure 5). The patient had no symptoms at this time and there were no signs of infection.

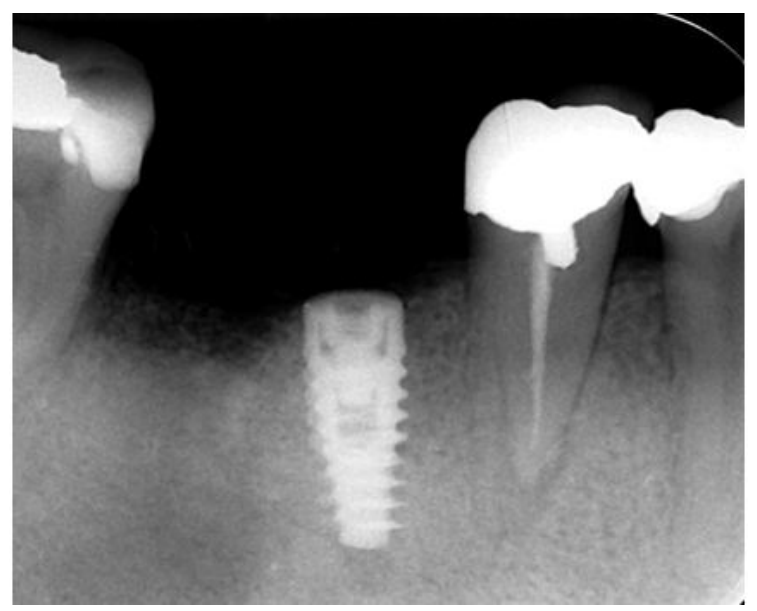

Figure 5: A new implant in different location after a month

\section{Discussion}

Acute apical peri-implantitis (AAPI) is an acute painful inflammation in the apical region of the implant. Different factors may lead to AAPI. One of the complicated reasons of AAPI is placed implant after extract a teeth with history of infection. Our patient had a tooth which had infection. 
In terms of diagnosis and treatment, acute apical peri-implantitis (AAPI) is more destructive than sub-acute apical peri-implantitis. The AAPI, unless diagnosed and treated early, may lead to implant failure in the first weeks after its placement.

An AAPI is a severe inflammatory response to microorganisms or their irritants. According to the authors, despite the curettage of the sockets and the prolonged waiting time until implant insertion, bacteria could remain in the bone causing subsequent development of lesions in the implants [7]. Our case supports the findings of the literature. Socket curettage was performed in the patient, but AAPI occurred three weeks later.

In the AAPI form, the lesion is symptomatic. It is diagnosed with the presence of a radiolucency around the apex of the implant, pain, gingival redness, painful soft swollen mucosa and, suppuration or fistula. There are slightly swelling in right cheek and pain in our patient. In the periapical radiograph revealed periapical radiolucency around the implant. But, this implant was immobile. There was not periodontal pathologic pocket the implant around.

The correct diagnosis of these lesions in their early stages allows their early treatment, and prevents implant failure. In a patient presenting with acute pain, well localized in relation to the apex of the implant, after a short period (1 to 3 weeks) since implant placement, presence of an acute apical peri-implantitis (suppurated or non-suppurated, depending on the existence of apical radiolucency or not) must be suspected and surgical treatment should be performed (implant apical surgery) [16]. Periapical radiolucency may sometimes be casual findings during routine radiographic assessments. If the patient is asymptomatic and the diameter of the radiolucent area is small, it is not necessary to treat the lesion; over preparation of the implant bed is the most probable cause, and only periodic radiographic controls should be done. If, in any of the controls, the radiolucency has increased in size or the patient develops symptoms, the surgical treatment will be applied.

In the subacute stage, the symptoms are less marked and there is bone destruction. The apical radiolucent area may be accompanied by bone destruction around the implant body, and soft tissue signs such as a fistulous tract or a vestibular abscess may be present. In these cases we must ensure that the stability of the implant has not been damaged; if the implant is mobile, it must be extracted, and if not, implant apical surgery must be performed [16].

The most studied treatment of implant periapical lesions with no associated implant mobility is implant apical surgery [16]. The most authors also, curettaged the lesion and irrigated with solution $[3,7,17]$. There are several agents for decontamination of the implant surface, such as air-powder abrasive technique, $3 \%$ hydrogen peroxide, chlorhexidine, citric acid or tetracycline pastes [7,17-20]. Chlorhexidine is considered the most efective agent for decontamination of the implant surface [9,21].

Sometimes, bone regeneration materials are used, accompanied or not with tissue regeneration barriers, in order to achieve complete bone regeneration of the defect [3,22]. Other authors [7,20,23], suggest sectioning the implant apex in those cases in which total removal of the granular tissue is not assured, and when working within the maxillary sinus or nasal cavity. Scarano et al. [8] decided to remove the implant in a patient because of pain persistence after treatment with analgesics; Oh et al. [24] removed one implant which presented mobility, and Sussman [25] recommended to remove the implant in all cases to prevent the onset of osteomyelitis.

Waasdrop and Reynolds [26] suggested that asymptomatic implant periapical lesions could be resolved by antibiotic therapy without surgical intervention, as a lesion of this type was fully resolved after treatment with antibiotics (amoxicillin 500 mg three times a day during 10 days). According to these authors the apical radiolucency presented by their patient was caused by an over preparation of the implant bed, and the patient never showed signs of infection or inflammation; thus the diagnosis of apical periimplantitis is uncertain. Other authors consider that in the presence of inactive or asymptomatic lesions treatment is not indicated [13]. According to some published case series [17,23], initial treatment with antibiotics was not effective to control symptomatic or active lesions, which required surgical access. Romanos et al. [27] concluded in their review that antibiotic treatment alone is not effective.

Romanos et al. [27] studied the prognosis of implant apical lesions after reviewing all cases published up to December 2007; 75\% of the implants diagnosed with periapical lesion survived after treatment, with follow-up periods ranging between 4 months and 7 years. Most studies reported few clinical cases, and it is difficult to determine the prognosis of implants treated with periapical surgery.

An AAPI form requires treatment to prevent bone resorbtion. The most adequate treatment of this pathology in the acute stage if there is no loss of implant stability is antibiotic treatment and then apical surgery. In the acute stage, if there is implant mobility, the extraction of the implant is necessary. The implant of our patient was not mobility but it was decided to remove his implant.

\section{Conclusion}

There are very different treatment approaches in acute apical peri-implantitis. In the acute apical peri-implantitis if there is no loss of implant stability, the correct treatment approach is implant periapical surgery.

\section{References}

1. McAllister BS, Masters D, Meffert RM (1992) Treatment of implants demonstrating periapical radiolucencies. Pract Periodontics Aesthet Dent 4: 37-41.

2. Sussman HI, Moss SS (1993) Localized osteomyelitis secondary to endodontic-implant pathosis. A case report. J Periodontol 64: 306-10. 
3. Quirynen M, Vogels R, Alsaadi G, Naert I, Jacobs R, et al. (2005) Predisposing conditions for retrograde peri-implantitis, and treatment suggestions. Clinc Oral Implants Res 16: 599-608.

4. Piattelli A, Scarano A, Balleri P, Favero GA (1998) Clinical and histologic evaluation of an active "implant periapical lesion": a case report. Int J Oral Maxillofac Implants 13: 713-6.

5. Chaffee NR, Lowden K, Tiffee JC, Cooper LF (2001) Periapical abscess formation and resolution adjacent to dental implants: clinical report. J Prosthet Dent 85: $109-12$.

6. Esposito M, Hirsch J, Lekholm U, Thomsen P (1999) Differential diagnosis and treatment strategies for biologic complications and failing oral implants: a review of the literature. Int J Oral Maxillofac Implants 14: 473-90.

7. Ayangco L, Sheridan PJ (2001) Development and treatment of retrograde peri-implantitis involving a site with a history of failed endodontic and apicoectomy procedures: a series of reports. Int J Oral Maxillofac Implants 16: 412-7.

8. Scarano A, Di Domizio P, Petrone G, Iezzi G, Piattelli A (2000) Implant periapical lesion: a clinical and histologic case report. J Oral Implantol $26: 109-13$.

9. Ramanauskaite A, Juodzbalys G, Tözüm TF (2016) Apical/Retrograde Periimplantitis/Implant Periapical Lesion: Etiology, Risk Factors, and Treatment Options: Systematic Review. Implant Dent 25: 684-97.

10. Thompson-Sloan TN, Kolhatkar S, Bhola M (2012) Management of Retrograde Peri-Implantitis by Apical Resection and Guided Bone Regeneration in Adjacent Maxillary Implants. Clin Adv Periodont 2: 250-5.

11- Villa R, Rangert B (2007) Immediate and early function of implants placed in extraction sockets of maxillary infected teeth: a pilot study. J Prosthet Dent 97: S96-S108.

12. Crespi R, Capparé P, Crespi G, Lo Giudice G, Gastaldi G, et al. (2017) Immediate Implant Placement in Sockets with Asymptomatic Apical Periodontitis. Clinic Implant Dent and Related Research 19: 20-7.

13. Reiser GM, Nevins M (1995) The implant periapical lesion: etiology, prevention, and treatment. Compend Contin Educ Dent 16: 768-72.

14. Peñarrocha Diago M, Boronat López A, Lamas Pelayo J (2006) Update in dental implant periapical surgery. Med Oral Patol Oral Cir Bucal 11: E429-32.

15. Misch CE (1999) Density of bone: effect on surgical approach and healing In: Contemporary Implant Dentistry (2 ${ }^{\text {nd }}$ Edn) St Louis, Mo: CV Mosby, USA.

16. Peñarrocha Diago M, Maestre Ferrín L, Cervera Ballester J, Peñarrocha Oltra D (2012) Implant periapical lesion: Diagnosis and treatment. Med Oral Patol Oral Cir Bucal 17: 1023-7.

17. Peñarrocha-Diago M, Boronat-Lopez A, García-Mira B (2009) Inflammatory implant periapical lesion: etiology, diagnosis, and treatment--presentation of 7 cases. J Oral Maxillofac Surg 67: 168-73.

18. Ataullah K, Chee LF, Peng LL, Lung HH (2006) Management of retrograde peri-implantitis: A clinical case report. J Oral Implantol 32: 308-12.

19. Chan HL, Wang HL, Bashutski J, Edwards PC, Fu JH, et al. (2011) Retrograde peri-implantitis: A case report introducing an approach to its management. J Periodontol 82: 1080-8.

20. Balshi SF, Wolfinger GJ, Balshi TJ (2007) A retrospective evaluation of a treatment protocol for dental implant periapical lesions: long-term results of $39 \mathrm{implant}$ apicoectomies. Int J Oral Maxillofac Implants 22: 267-72.

21. Ntrouka V, Hoogenkamp M, Zaura E, van der Weijden F (2011) The effect of chemotherapeutic agents on titanium-adherent biofilms. Clin Oral Implants Res 22: $1227-34$

22. Bretz WA, Matuck AN, de Oliveira G, Moretti AJ, Bretz WA (1997) Treatment of retrograde peri-implantitis: clinical report. Implant Dent 6: 287-90.

23. Dahlin C, Nikfarid H, Alsén B, Kashani H (2009) Apical peri-implantitis: possible predisposing factors, case reports, and surgical treatment suggestions. Clin Implant Dent Relat Res 11: 222-7.

24. Oh TJ, Yoon J, Wang HL (2003) Management of the implant periapical lesion: a case report. Implant Dent 12: 41-6.

25. Sussman HI (1998) Periapical implant pathology. J Oral Implantol 24: 133-8.

26. Waasdrop J, Reynolds M (2010) Nonsurgical treatment of retrograde peri-implantitis: a case report. Int J Oral Maxillofac Implants 25: 831-3.

27. Romanos GE, Froum S, Costa-Matins S, Meitner S, Tarnow DP (2011) Implant periapical lesions: Etiology and treatment options. J Oral Implantol 37: 53-63.

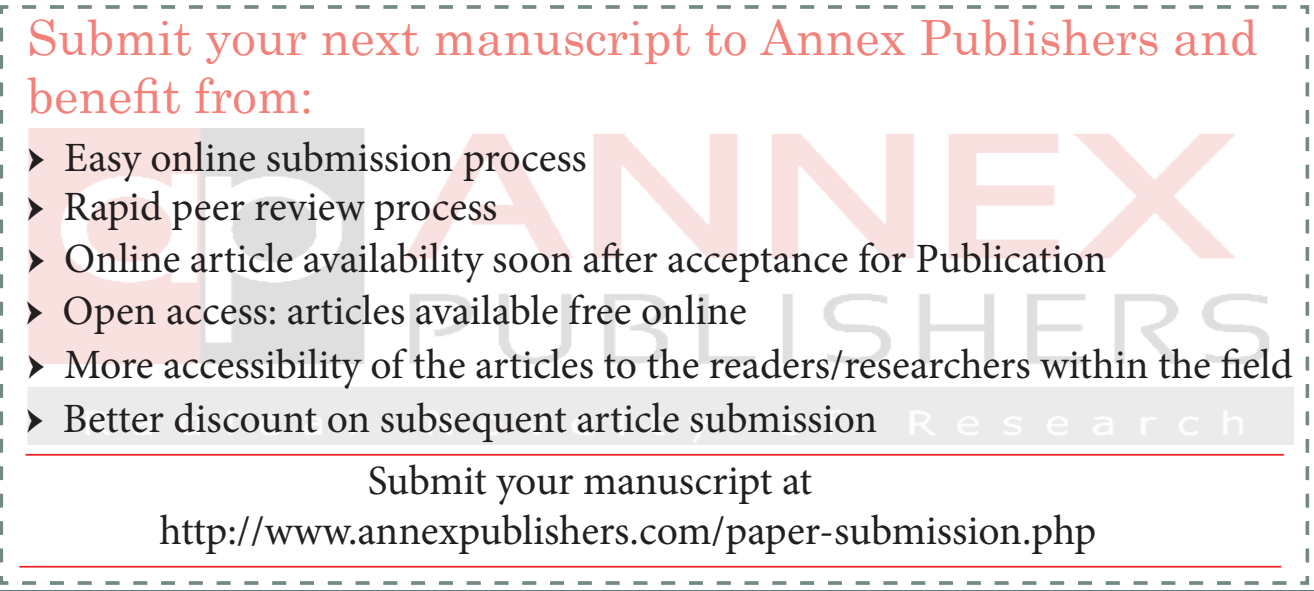

